\title{
TCP-based window-size delegation method for TXOP Exchange in wireless local area networks
}

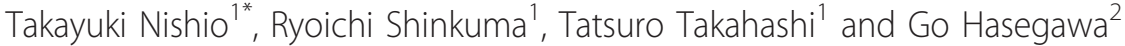

\begin{abstract}
We propose a TCP window-size delegation method for downlink TXOP (transmission opportunity) Exchange in wireless local area networks (WLANs). In our method, the 'compliant' stations (STAs) cooperatively use their available bandwidth in accordance with their throughput demand. We realize our method only with minimal modifications of the TCP functions of a proxy server, which lets one station (the TXOP provider) delegate TCP window size to another station (the TXOP client) so that the provider delegates its TXOPs in WLANs to the client. Our method enables an STA to flexibly delegate TXOPs to another STA without adversely affecting the legacy STAs, which is confirmed by computer simulations. We also confirmed that our method requires no modification to legacy access points and STAs.
\end{abstract}

Keywords: Bandwidth delegation, TCP window size, TXOP Exchange, Wireless local area networks

\section{Introduction}

In offices, homes, and public spaces, IEEE 802.11 wireless local area networks (WLANs) has been extensively used to provide wireless Internet connection services. In WLANs, stations (STAs) connected to an access point (AP) compete for transmission opportunities (TXOPs) using the carrier sense multiple access with collision avoidance (CSMA/CA). TXOPs are almost equally assigned to the STAs and the AP without considering each STA's throughput demand, especially when the traffic load is heavy. Although many quality of service (QoS) control mechanisms applicable for WLANs have been proposed including IEEE 802.11e, IntServ and DiffServ [1-3], they are not widely used basically because they require 'physical replacement' of existing APs or edge routers.

To solve this problem, we proposed TXOP Exchange [4-6]. In WLANs, the number of TXOPs obtained by each STA determines the throughput of the STA. Therefore, for the uplink, we modified only the STAside media access control (MAC) protocol so that the STAs participating in TXOP Exchange (compliant STAs) can directly delegate TXOPs without affecting the performances of the other STAs. In TXOP

\footnotetext{
* Correspondence: nishio@cube.kuee.kyoto-u.ac.jp

${ }^{1}$ Graduate School of Informatics, Kyoto University, Kyoto, Japan

Full list of author information is available at the end of the article
}

Exchange, compliant STAs cooperatively use their available bandwidth in accordance with their required throughputs. Consider an example of a wireless access network with one AP, two STAs compliant with our architecture, and other STAs. Each of the STAs is downloading a large file, like a video file or a zipped file of photos, to a storage server. The throughput of the STAs is saturated at $800 \mathrm{Kbps}$. Suppose that one of the compliant STAs, STA 1, wants to increase its throughput to $1 \mathrm{Mbps}$ to download its file faster. If the other compliant STA, STA 2, is not interested in downloading its file faster, then, as shown in Figure 1, it can delegate some of its TXOPs to STA 1 so that the throughput of STA 1 increases up to $1 \mathrm{Mbps}$. Throughput increase is always beneficial for STAs regardless of how much it is particularly when they download files or receive audio/ video streams with progressive download. In this example, STAs 1 and 2 are a TXOP client and a TXOP provider, respectively. Each compliant STA can become a provider or a client as necessary. Thus, cooperative exchange of TXOPs enables STAs to satisfy the throughput requirements each other. TXOP Exchange enables compliant STAs to flexibly exchange bandwidth without modifications on the legacy APs and without affecting the performances of the other "non-compliant" STAs, as illustrated in Figure 2.

\section{SpringerOpen ${ }^{\odot}$}

(C) 2011 Nishio et al; licensee Springer. This is an Open Access article distributed under the terms of the Creative Commons Attribution License (http://creativecommons.org/licenses/by/2.0), which permits unrestricted use, distribution, and reproduction in any medium, provided the original work is properly cited. 


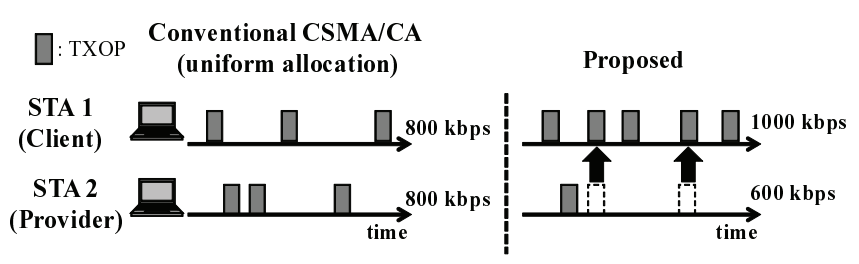

Figure 1 TXOP delegation in CSMA/CA.

We previously focused on the use of TXOP Exchange for the uplink $[4,5]$. In this paper, we focus on extending TXOP Exchange so that it can be used for the downlink as well. Unlike the uplink, we cannot realize TXOP Exchange for downlink only by MAC-layer modification at STAs since the packets are sent to the STAs from the connecting AP by using the AP's TXOP; in other words, the STAs share TXOPs of the AP for their downlink communications. On the other hand, AP sends a packet in the top of sending queue of the AP. Therefore, to enable an STA to delegate its TXOPs to another STA, we need to control the number of packets in the AP sending queue. Considering the implementation constraint and cost, we propose a transport-level control method that uses proxy servers to control the number of packets arriving at the AP. A proxy server plays roles of a coordinator between STAs and a manager for TCP connections of compliant STAs. In this method, a TXOP provider delegates a chance to increase its TCP window size to the TXOP client, which is controlled by the proxy server. The proxy server also decreases the window size for the TXOP provider where as that for the TXOP client would be decreased with the legacy TCP. This method does not require any modifications of the APs or the legacy STAs and is applicable to access networks other than WLANs since it is based on end-to-end TCP-level controls.

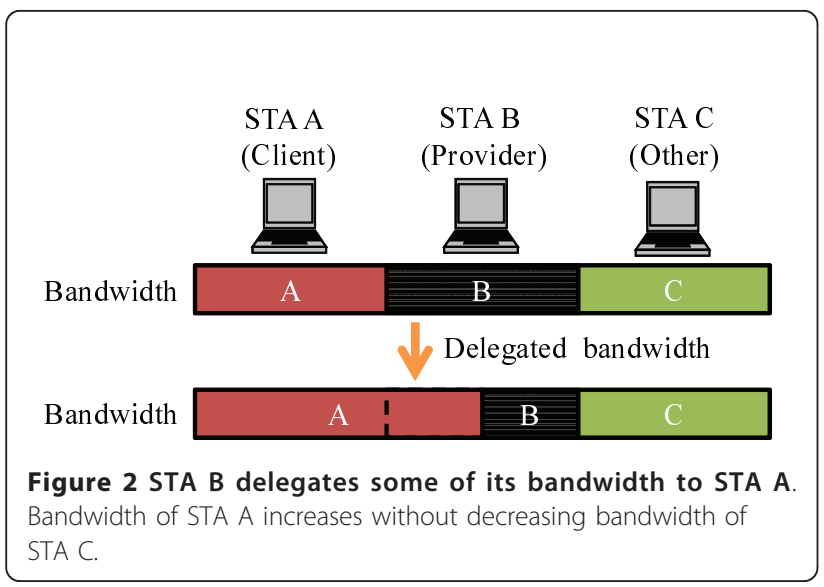

The rest of this paper is organized as follows. Section 2 briefly introduces our TXOP delegation method for the uplink and show how it works. In Section 3, we describe out TXOP delegation method for the downlink in detail. In Section 4, we observe how our method works through computer simulations and verify that it enables an STA to delegate throughput to another STA, while the other STAs see the same throughput as before. We also discuss the performance with varying the number of other STAs and round trip time (RTT). Finally, we mention the related work and conclude our paper in Sections 5 and 6.

\section{Previous work: TXOP Exchange for uplink}

CSMA/CA, which is deployed in WLANs, was originally designed for assigning TXOPs equally to the STAs when the traffic load is heavy. An STA is able to send a data frame when it obtains a TXOP. In WLANs, to the best of our knowledge, there has been no method that enables an STA to delegate its TXOPs to another STA without modification to APs. Therefore, we newly designed a TXOP delegation method in WLAN in [4]. The proposed method requires small modification to the conventional IEEE 802.11 mechanism only of compliant STAs and it does not affect co-existing legacy STAs' behaviors. The proposed method exploits RTS/ CTS mechanism to provide such characteristics.

In CSMA/CA with RTS/CTS, an STA sends an RTS frame to the AP before sending a data frame, and the AP sends a CTS frame back to the STA to allocate a TXOP to it and to prohibit the other STAs from sending any frames during the network allocation vector (NAV) period. Since the RTS frame includes the sender address field, the AP can recognize which STA sent it. In our TXOP Exchange for uplink, the TXOP provider replaces the source address field in the RTS frame with the client address to force the AP to allocate a TXOP to the client with the corresponding CTS frame. We call this RTS frame the coop RTS frame. The provider calculates the NAV duration based on the data frame size and the physical transmission rate of the client and includes it in the coop RTS frame. We also introduce a 
coordination server, which a proxy server plays a role of as mentioned in Section 1, to handle the client's informations, such as MAC and IP address, physical transmission rate, data frame size, and throughput requirement. As discussed in our prior work [6], the coordination server works to ensure fairness between STAs and stimulate them to cooperate each other. We also proposed an algorithm that determines how frequently the coop RTS frames are sent from the provider according to the required throughput of the client $[4,5]$.

We implemented this method in the QualNet 4.5 [7] simulator. Figure 3 shows the average throughput of each STA as a function of $N_{o}$, where is a single-AP network with a provider, client, and $N_{o}$ other STAs. We assume that the client is requesting additional $500 \mathrm{Kbps}$ throughput from the current throughput. This figure shows that the provider can provide $500 \mathrm{Kbps}$ from its throughput to the client. For example, when the number of other STAs was twelve, the throughput before TXOP delegation (conventional in the figure) was $1.05 \mathrm{Mbps}$, while the throughputs for the provider and the client after delegation were 0.61 and 1.53 Mbps. We should note that the other STAs in our method behaved the same as in the 'conventional', which indicate the throughput when STAs do not exchange TXOPs.

\section{TXOP Exchange for downlink}

In the downlink of WLANs, the throughput for each STA connected to the AP depends on the number of packets for each STA in the AP sending queue, because the AP sends the packet at the top in its sending queue when it obtains a TXOP. We here consider an example of a wireless access network with one AP and three STAs, STA A, STA B, and STA C. If the ratio of the number of packets for STA A, B and C in the sending queue of AP is $a, b$, and $c$, the downlink throughputs of the STAs are $\theta_{A}=a \cdot \theta^{\text {total }}, \theta_{B}=b \cdot \theta^{\text {total }}$, and $\theta_{C}=c$. $\theta^{\text {total }}$, respectively, where $\theta^{\text {total }}$ is the total throughput of STAs. Therefore, for instance, if we want to assign a portion of the bandwidth for STA B to STA A without affecting STA $C$ as illustrated in Figure 2, $a$ and $b$ should be increased and decreased, respectively, while maintaining $c$ constant. Therefore, we enable the throughput delegation by controlling the number of packets for each STA in the AP sending queue.

There are a couple of possible ways of controlling the ratio of the number of packets for STAs in the AP sending queue. One solution may be to replace every AP by the ones equipped with a new queueing function, which is a counter direction of our goal. We therefore chose a transport-level approach. Assigning multiple TCP flows to an STA can be another solution to increase throughput for the specific STA. However, as we will show a simulation example in Figure 4, it is impossible to control throughputs without affecting other STAs. Therefore, we consider modifying the transport protocol in this paper. Since it is unrealistic to modify the transport protocol for every server, we assume that proxy servers are located in each autonomous system (AS) network between the STAs and corresponding servers from which STAs are originally downloading data. The detail of our approach is described in the next subsection.

\subsection{System model}

Figure 5 illustrates the system model of our TXOP Exchange for the downlink. A proxy server is located in each AS network, while our compliant STAs maintain sessions with the proxy server and download data from

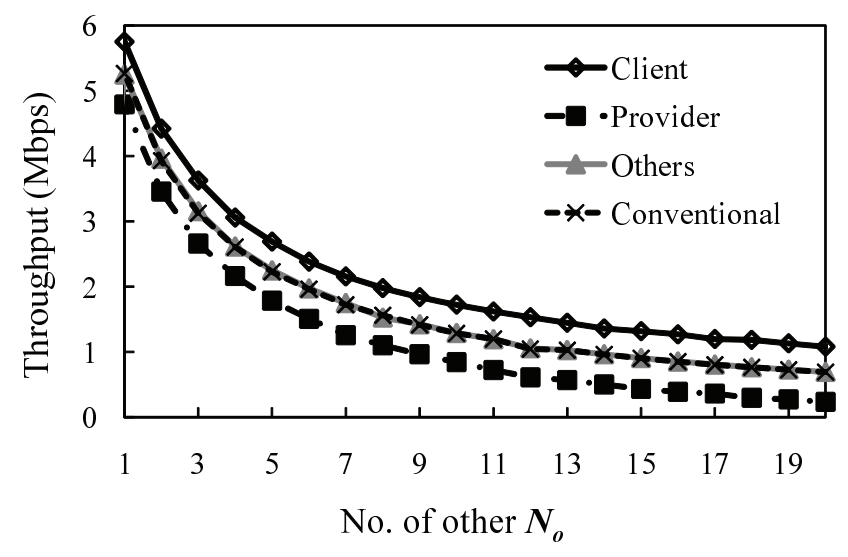

Figure 3 Average throughput of each STA versus No. of other STAs, $N_{o}$, in a network with provider, client, and other $N_{o}$ STAs, each of which sends saturated UDP flow. QualNet 4.5 simulator was used; physical transmission rates were fixed to 24 Mbps; provider, client and other STAs send saturated UDP with 1,500 bytes of data. 


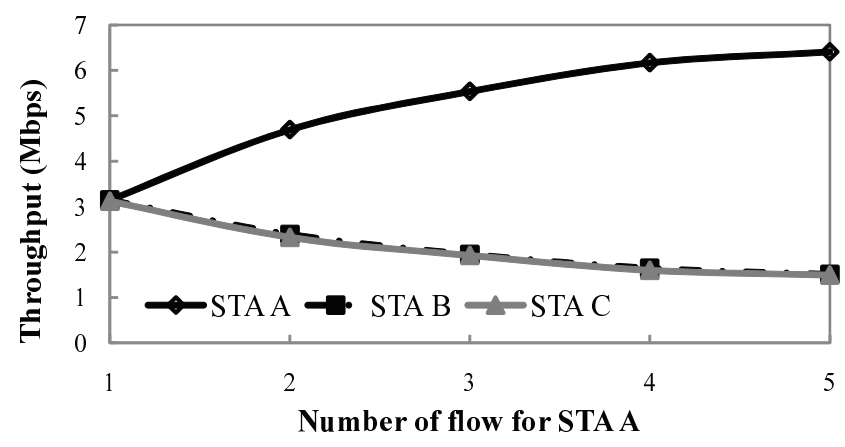

Figure 4 Throughputs for STAs A, B, and C versus the number of flows assigned to STA A. We used the model in Figure 9.

the corresponding servers via the proxy server. Here, we consider an example of window-size delegation between a pair of a provider and a client, both of which connect to an AP. Since the number of packets sent by a server to an STA in RTT is approximately the same as the window size of a TCP flow for the STA [8], the proxy server changes the provider's and client's window sizes while keeping the total size unchanged so as to control the ratio of the numbers of their packets arriving at the AP but keeping the number of packets for other STAs unchanged. The proxy server manages TCP flows for the two STAs to enable the provider to delegate its TCP window size to the client. Since TCP flows from the corresponding servers are terminated at the proxy server, what we need to discuss is only the behaviors of TCP flows from the proxy server to the STAs.

\subsection{Basic mechanism of TCP window-size delegation}

Figure 6 represents an example of behaviors of TCP window sizes for a provider, a client, and a non-compliant STA when our window-size delegation works ideally. When the proxy server has received a normal ACK packet, which differs from triple-duplicate ACKs, from the provider, in other words, when the flow for the provider is in the congestion-avoidance phase, the proxy server increases either of the window sizes for the client $W_{c}$ or for the provider $W_{p}$ as below:

$$
\begin{aligned}
& W_{p}=W_{p}+\Delta_{p}(\text { with prob. } 1-\alpha) \\
& W_{c}=W_{c}+\Delta_{p}(\text { with prob. } \alpha)
\end{aligned}
$$

where $\Delta_{p}$ was set equal to $1 / W_{p}$ as in the conventional TCP. It should bonoted that, when the proxy server has received a normal ACK packet from the client, just as in the conventional TCP, the proxy server updates the client's window size as $W_{c}=W_{c}+\Delta_{c}$, where $\Delta_{c}=1 / W_{c}$.

The algorithm in Equation 1 means that as $\alpha$ increases, more window size is delegated from the provider to the client. When the flow for the client is in the slow-start phase and when the flow for the provider is in congestion-avoidance phase, the increase in the window size is kept held until the flow for the client comes back in the congestion-avoidance phase and then will be added to the client's window size. In addition, the proxy server records and updates a virtual window sizes of the provider and client, $W_{p}^{v}$ and $W_{c}^{v}$, respectively, which they would obtain if the provider did not delegate its window size to the client. The virtual window size, $W_{x}^{v}(x=\operatorname{cor} p)$,

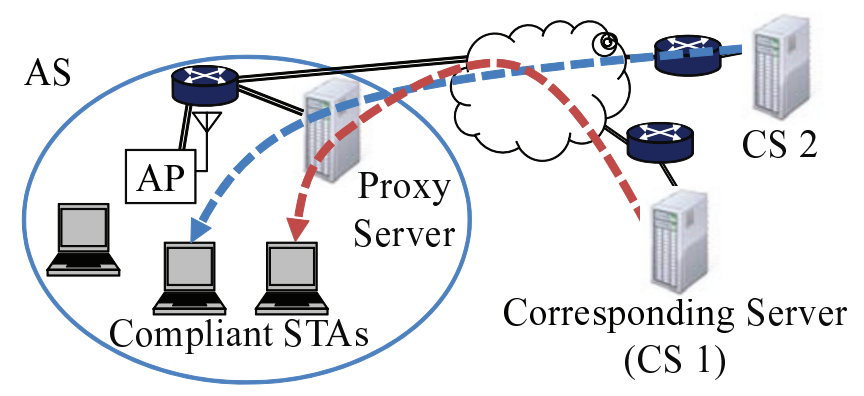

Figure 5 System model of downlink TXOP Exchange. Compliant STAs download data via proxy server. 


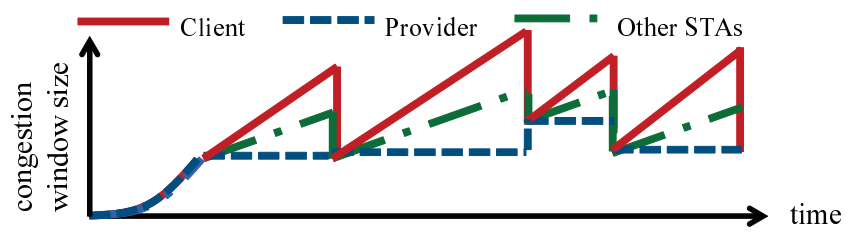

Figure 6 Ideal change in congestion window size over time when $\alpha=1.0$

is updated by $W_{x}^{v}=W_{x}^{v}+1 / W_{x}^{v}$ when $W_{x}^{v}>0$ and $W_{x}^{v}=W_{x}+1 / W_{x}$ when $W_{x}^{v}=0 . W_{x}^{v}$ is updated only when the proxy server receives an ACK packet from the STA $x$.

Although the above procedure enables a client to increase its window size, its throughput does not necessarily increase because the larger window size of a TCP connection may increase a loss ratio of data packets it sends. Therefore, when the proxy server receives tripleduplicate ACKs from a client, it decreases the provider's and client's window size as below:

$$
\begin{aligned}
& W_{p}=T h_{p}^{s s}=W_{p}^{v} / 2(\text { with prob. } \quad \beta) \\
& W_{c}=T h_{c}^{s s}=W_{c}^{v} / 2(\text { with prob. } 1-\beta),
\end{aligned}
$$

where $T h_{p}^{s s}$ and $T h_{c}^{s s}$ are the slow-start threshold of the flow for the provider and client, respectively. Equation 2 means that, as $\beta$ increases, $W_{c}$ decreases less often, which implies the client's window size is maintained larger. It should be noted that the proxy server retransmits the packets corresponding to the duplicate ACKs in both cases in Equation 2. After decreasing the window size of the client/provider, the virtual window size of the client/provider is initialized by 0 .

\subsection{How to set $\alpha$ and $\beta$}

Figure 7 shows the state diagram of our window-size delegation method. In our method, first of all, a proxy server is required to set $\alpha$ and $\beta$ in accordance with required throughput and other referenced parameters. Then, it starts the window-size delegation. In this section, we discuss how to set $\alpha$ and $\beta$.

3.3.1 $\alpha$

Here, we consider a case where STA $i$ becomes a client, STA $j$ becomes a provider, respectively. We set optimal $\alpha$ in accordance with a required throughput of STA $i \theta_{i}^{r}$ and a 'referenced' throughput of the STA $\theta_{i}$. As the first step just after the 'start' in Figure 7, a proxy server measures $\theta_{i}$ before starting the window-size delegation, and then the proxy server calculates appropriate $\alpha$ from $\theta_{i}$ and $\theta_{i}^{r}$.
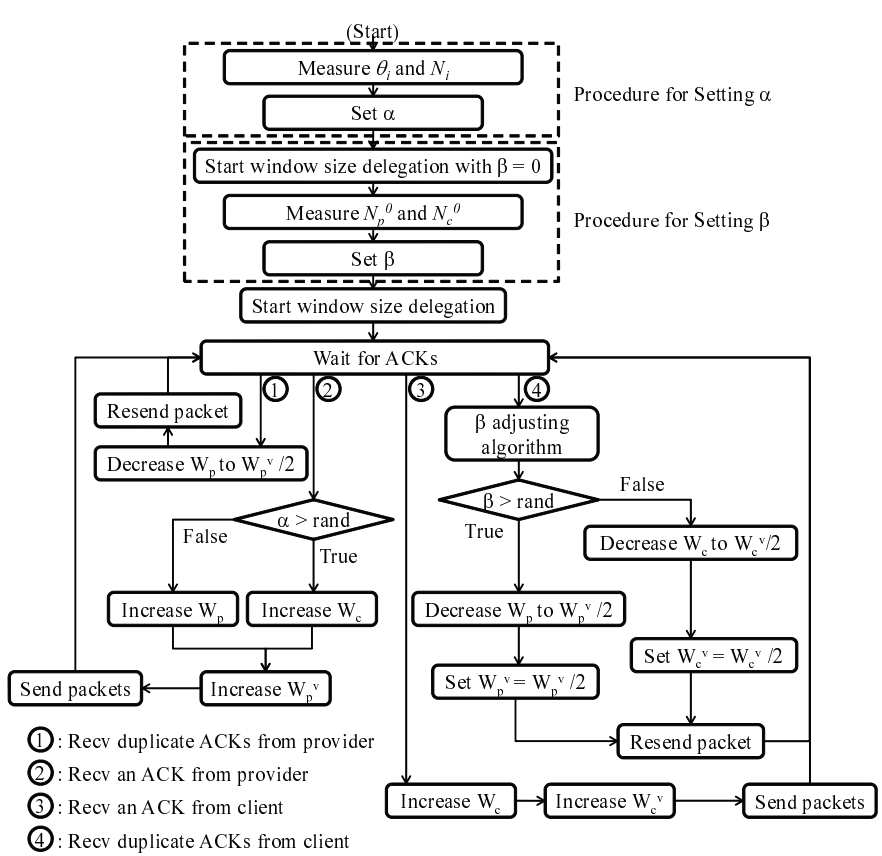

Figure 7 States diagram of window-size delegation. 
Let us show the relationship between throughput and window size without window-size delegation. Ideally, in the steady state, the congestion window size of STA $i$ $W_{i}(t)$ changes as the dashed line does in Figure 8. In this case, the relationship is written as

$$
\begin{aligned}
\theta_{i}=\left(D_{i}\right) \frac{\int_{T D_{i}} W_{i}(t) \mathrm{d} t}{T D_{i}} & =\left(D_{i}\right) \frac{\frac{W_{i}^{d}}{2} \cdot T D_{i}+\frac{1}{2} \cdot \frac{W_{i}^{d}}{2} \cdot T D_{i}}{T D_{i}} \\
& =\left(D_{i}\right)\left(\frac{3}{2} \cdot \frac{W_{i}^{d}}{2}\right)
\end{aligned}
$$

where $D_{i}$ is the size of packets sent to STA $i, W_{i}^{d}$ is the congestion window size when triple-duplicate ACKs occur in the steady state; $T D_{i}$ is a duration after tripleduplicate ACKs occur until the next triple-duplicate ACKs occur.

When STA $i$ becomes a client, if our method works ideally, the congestion window size of STA $i W_{c i}(x)$ changes as the gray line does in Figure 8. Here, the throughput of STA $i \theta_{c i}$ is written as

$$
\begin{aligned}
& \theta_{c i}=\left(D_{i}\right) \frac{\int_{T D_{i}} W_{c i}(t) \mathrm{d} t}{T D_{i}}=\left(D_{i}\right) \frac{\frac{W_{i}^{d}}{2} \cdot T D_{i}+\frac{1}{2}\left(\frac{W_{i}^{d}}{2}+\Delta\right) \cdot T D_{i}}{T D_{i}} \\
& =\frac{3+\alpha}{3}\left(D_{i}\right)\left(\frac{3}{2} \cdot \frac{W_{i}^{d}}{2}\right) \\
& =\frac{3+\alpha}{3} \cdot \theta_{i},
\end{aligned}
$$

where $\Delta=\alpha \cdot W_{i}^{d} / 2$ in Equation 4. In Figure 8, window-size delegation increases an increasing slope of $W_{c i}$ $(x)$ in an interval of linear to $(1+\alpha)$-fold from $W_{i}(x)$. If we want to control window-size delegation so that $\theta_{c i}$ equals to $\theta_{i}^{r}$, from Equation $4, \alpha$ should be set as

$$
\alpha=\frac{3 \theta_{i}^{r}}{\theta_{i}}-3
$$

\subsection{2 $\beta$}

For setting $\beta$, we use $N_{c i}^{0}$ and $N_{p j}^{0}$, which are how many times triple-duplicate ACKs for a client and a provider, respectively, occur while the window-size delegation is operated with $\alpha$ given in Section 3.3.1 and $\beta=0$. After setting $\alpha$, a proxy server starts window-size delegation with the $\alpha$ and $\beta=0$ and then measures $N_{c i}^{0}$ and $N_{p j}^{0}$, as illustrated in Figure 7. Then, $\beta$ is set using measured parameters, $N_{c i}^{0}$ and $N_{p j}^{0}$.

How many times the proxy server decreases the window sizes for the client and the provider, $N_{c i}^{d} N_{p j}^{d}$, can be approximated by $N_{c i}^{d}=(1-\beta) N_{c i}^{0}$ and $N_{p j}^{d}=N_{p j}^{0}+\beta \cdot N_{c i}^{0}$, respectively. The effect on the other STAs is minimized when $N_{c i}^{d}=N_{p j}^{d}$ because the total of the decreased window size caused by triple-duplicate ACKs for the provider and the client is almost twice as that for the other non-compliant STA. Therefore, $\beta$ should be set to $\left(N_{c i}^{0}-N_{p j}^{0}\right) / 2 N_{c i}^{0}$.

\subsection{Adjusting algorithm for $\beta$}

We discussed how to set $\beta$ in Section 3.3.2. However, even using the determined $\beta$, window-size delegation slightly affects throughputs of other STAs because of a difference of $N_{c i}$, which is how many times triple-duplicate ACKs occur in a certain period, from $N_{c i}^{0}$, which is initially measured. Here, if the probability that packet loss occurs is the same for all STAs in the network, the following relationship is obtained from Equation 4, which represents the relationship between the actual and referenced throughput of the client:

$$
N_{c i}=\frac{3+\alpha}{3} N_{i}
$$

where $N_{i}$ is the referenced number of how many times triple-duplicate ACKs occur in Figure 7. Assuming that

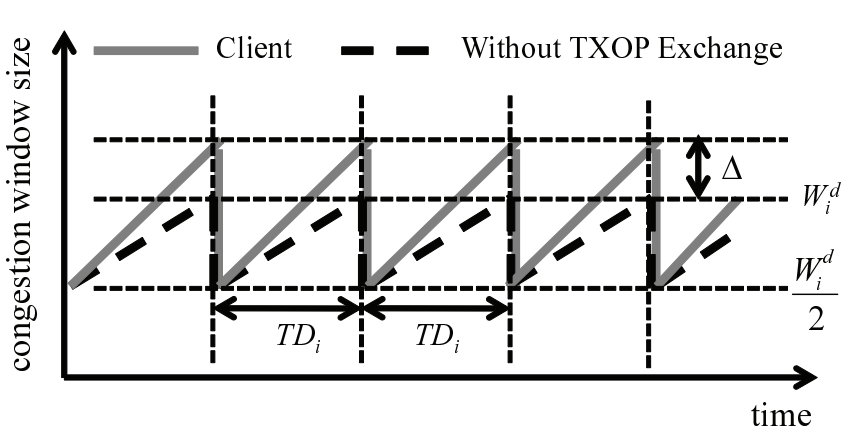

Figure 8 Ideal change of congestion window size in steady state. 
our window-size delegation method works ideally, if a provider delegates its window size to a client without affecting on others' throughputs, Equation 7 should be satisfied.

Based on the above discussion, we come up with $\beta$ adjustment algorithm that adjusts $\beta$ to maintain Equation 7 satisfied. A simple algorithm for this is

$$
\begin{aligned}
& \beta=\beta+\Delta^{u}\left(\text { if } N_{c i}<\frac{3+\alpha}{3} N_{i}\right) \\
& \beta=\beta-\Delta^{d}\left(\text { if } N_{c i}>\frac{3+\alpha}{3} N_{i}\right)
\end{aligned}
$$

The proxy server adjusts $\beta$ based on the algorithm every time duplicate ACKs are received from for STA $i$, which is illustrated in Figure 7. We set $\Delta^{u}=\Delta^{d}=1$ in the following simulation section to let $N_{c i}$ converge to $N_{i}(3+\alpha) / 3$ fast.

\section{Simulation evaluation}

\subsection{Simulation setup}

We evaluate our delegation method using QualNet simulator [7]. We assume a network in which a provider and a client download data using TCP from a corresponding server, while the other STA download data directly from another corresponding server. Each of the STAs used only one flow. We assume that a bandwidth between the proxy server and the corresponding server for the provider and the client is large enough not to limit throughputs for them. We also idealize wireless channels to make our discussion simple. For instance, when an STA with lower channel quality delegates its TXOPs to another STA with higher channel quality, the overall transmission efficiency might improve. This issue should be included in future work.
In Section 4.2, we will first observe the dependence of throughputs on $\alpha$ and $\beta$ of our method introduced in Section 3.2. To observe the basic characteristics of the proposed method, we used the network model illustrated in Figure 9, where the wireless link is modeled as simply a wired $10 \mathrm{Mbps}$ bottlenecklink; since every packet in a WLAN downlink is always sent from the AP, results from this model would not be far from the ones from a model with CSMA/CA. Therefore, here, the maximum total amount of throughputs of STAs is limited to $10 \mathrm{Mbps}$ by this bottlenecklink.

However, in Section 4.3, we will evaluate the effectiveness and the scalability of our method with a wireless network in a WLAN access link is the bottleneck as illustrated in Figure 10. The parameters regarding the wireless link completely follow the standard of IEEE 802.11a, though we fixed the transmission rates to $24 \mathrm{Mbps}$ with no channel errors. In this model, because of the MAC overhead, the total amount of throughputs of STAs is limited to approximately $13.5 \mathrm{Mbps}$ when there are three STAs.

\subsection{Dependence of throughputs on $\alpha$ and $\beta$}

We first demonstrate how a conventional method works. As mentioned in Section 3, a simple way to increase throughput of an STA is to assign multiple flows for the STA. Figure 4 shows the throughputs of each STA as a function of the number of flows assigned to STA A. With increase the number of flows for STA A, the throughput for STA A increases, while throughputs for STAs B and C decrease. As shown in this example, the conventional method may differentiate throughputs but it affects the non-compliant STAs significantly.

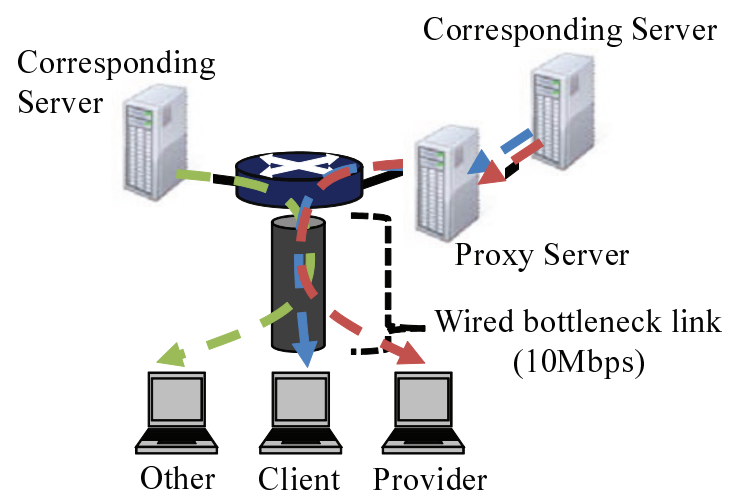

Figure 9 Simulation model. We used QualNet 4.5.1; TCP SACK with delay-ACKs, Nagale algorithm, RFC1323, and Keepalive probes; maximum segment size was 1,500 bytes; send buffer size was 65,000 bytes; receive buffer size was 65,000 bytes; wired bottlenecklink bandwidth was 10 Mbps, while other links bandwidth were $100 \mathrm{Mbps}$. 


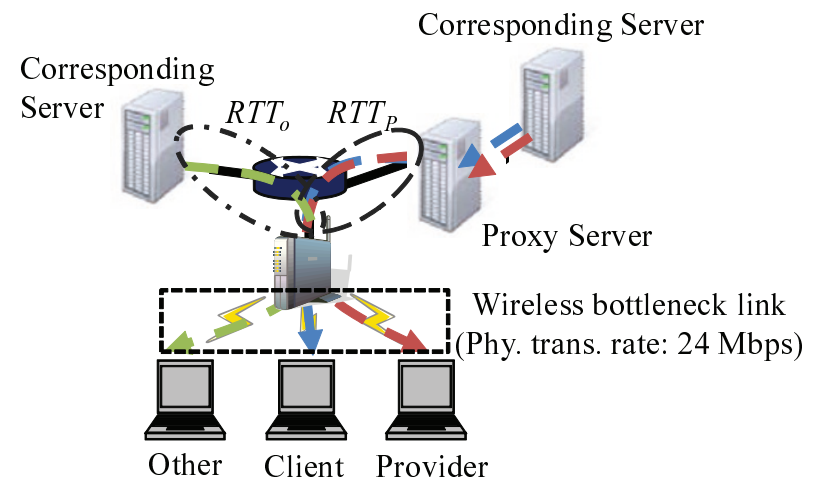

Figure 10 Simulation model of WLAN. We used QualNet 4.5.1; TCP SACK with delay-ACKs, Nagale algorithm, RFC1323, and Keepalive probes; maximum segment size was 1,500, bytes; send buffer size was 65,000 bytes; receive buffer size was 65,000, bytes; Access network is IEEE 802.11a WLAN with physical transmission rate of $24 \mathrm{Mbps}$; other links' bandwidth were $100 \mathrm{Mbps}$; RTp was $10 \mathrm{~ms}$ while RTTo varied from 10 to $500 \mathrm{~ms}$.

We next observe how our window-size delegation work with varying given $\alpha$ and $\beta$, which means, here, we did not use our criteria for determining $\alpha$ and $\beta$ described in Sections 3.3. Figures $11 \mathrm{a}$, b plots the throughput of each STA as a function of $\beta$ for $\alpha=0.3$ and 1.0, respectively. 'w/o delegation' means the average throughputs for STAs when our method is not used. As you first see in these figures, we successfully increased the throughput for the client compared with 'w/o delegation' by the delegation from the provider. We also observe the increased and decreased throughputs for the client and the provider increase, as given $\beta$ increases.

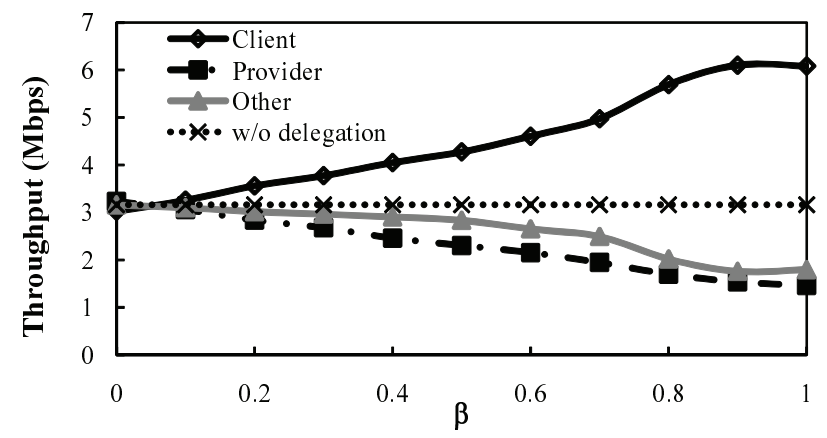

(a) $\alpha=0.3$

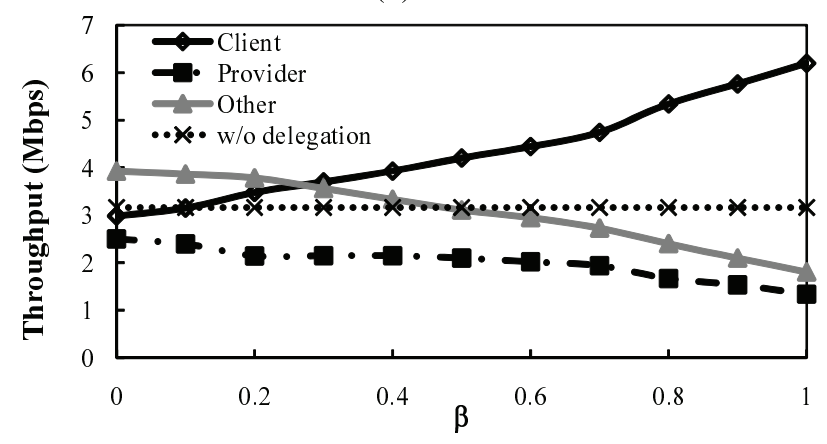

(b) $\alpha=1.0$

Figure 11 Throughputs for the client and the provider versus $\beta$ when a $\alpha=0.3, b \alpha=1.0$ with one additional TCP flow other than client and provider (other). We used the model in Figure 9. 
However, the throughput for the other STA is not kept equal to the one in 'w/o delegation' and depends on $\alpha$ and $\beta$. We see the intersection of the throughput for the other between with and without delegation at a combination of $\alpha$ and $\beta$, which suggests we have to choose $\alpha$ and $\beta$ appropriately.

Figures 12a, b, c show the throughput of each STA as a function of $\alpha$ for $\beta=0.3,0.5$, and 1.0, respectively. We can observe from these figure that the throughput of the client remains almost unchanged and independent of $\alpha$. However, the throughput for the other STA increased when $\alpha$ exceeded a certain point. Unless $\beta$ is 0.7 , when $\alpha$ was appropriately set, the throughput for the other STA was the same as w/o delegation.

The above results suggest we have to introduce an adaptive algorithm for determining $\alpha$ and $\beta$ appropriately. Now, we start to use our designed algorithm

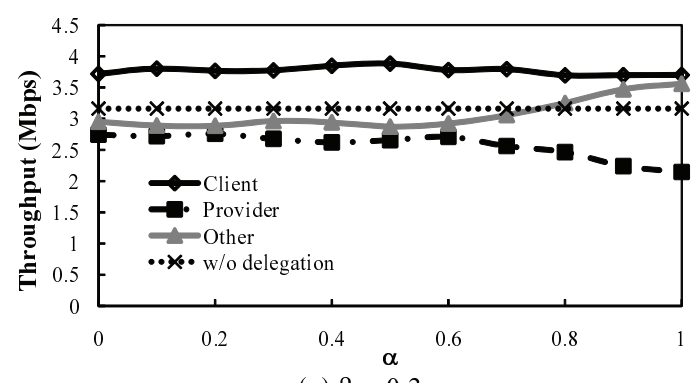

(a) $\beta=0.3$

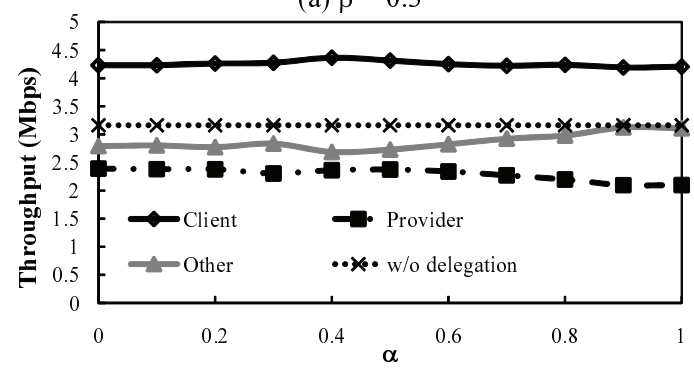

(b) $\beta=0.5$

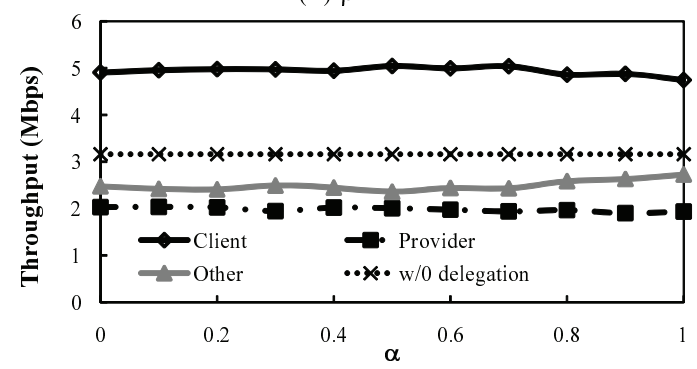

(c) $\beta=0.7$

Figure 12 Throughput for each STA versus $\alpha$ when a $\beta=0.3$, b $\beta=0.5, \mathrm{c} \beta=1.0$ with one additional TCP flow other than client and provider (other). The model in Figure 9 was used. described in Section 3.3. Figure 13 plots the result as a function of $\Delta_{r}$, which is the required additional throughput from the client: how much the client wants to increase its throughput than before starting TXOP Exchange. We see in the figure that the throughput of the client is successfully controlled almost equally to $\Delta_{r}$ without affecting that of the other STA.

\subsection{Performance of window-size delegation method in WLANs}

We here evaluate the performance of our method with using a wireless network in Figure 10. Figure 14 shows the average throughput of each STA as a function of $\Delta_{r}$. In this figure, we confirm the throughput for the client is increased almost equally to $\Delta_{r}$ compared with 'w/o delegation' without affecting the throughput for the other STA.

Next, we evaluate the scalability of our method through the following two scenarios, in which the same simulation parameters are used as in Figure 11 except the number of STAs and RTTs between corresponding servers and the AP.

First, we investigate a case where the number of other STAs varies. We assume a network with a provider, a client, and $N_{o}$ other STAs. RTTs here were identically set to $10 \mathrm{~ms}$. Figure 15 plots the average throughput of each STA as a function of $N_{o}$, in which 'w/o delegation (compliant)' indicates the throughput of compliant STAs which download data from the proxy server without the window-size delegation, while 'w/o delegation (other)' indicates the throughput of other STAs which download data from their corresponding server without the window-size delegation. We fixed the required throughput $\Delta_{r}$ to $300 \mathrm{Kbps}$. As seen in the figure, regardless of $N_{o}$, the client keeps its increased throughput approximately $300 \mathrm{Kbps}$ larger than that in 'w/o delegation (compliant)'. We also see the throughputs of the other STAs did not change from 'w/o delegation (others)'.

Second, we investigate a case where RTT between the corresponding server for other STAs and the AP RTT is different from RTT between the proxy server and the AP $R T T_{P}$. Since the proxy server is located in the same as the AP and the others' corresponding server is located in somewhere in the Internet, $R T T_{o}$ can be a little or much larger than $R T T_{P}$. We assume that $R T T_{P}$ was $10 \mathrm{~ms}$ while $R T T_{o}$ varied from 100 to $500 \mathrm{~ms}$. We fixed the total number of STAs to 10, which consists of a provider, a client and eight other STAs. Figure 16 shows average throughputs of each STAs versus RTT of flows for others. We set the required throughput $\Delta_{r}$ to $600 \mathrm{Kbps}$. This figure also shows that the client maintains its increased throughput approximately $600 \mathrm{Kbps}$ larger than compared with 'w/o delegation (compliant)' 


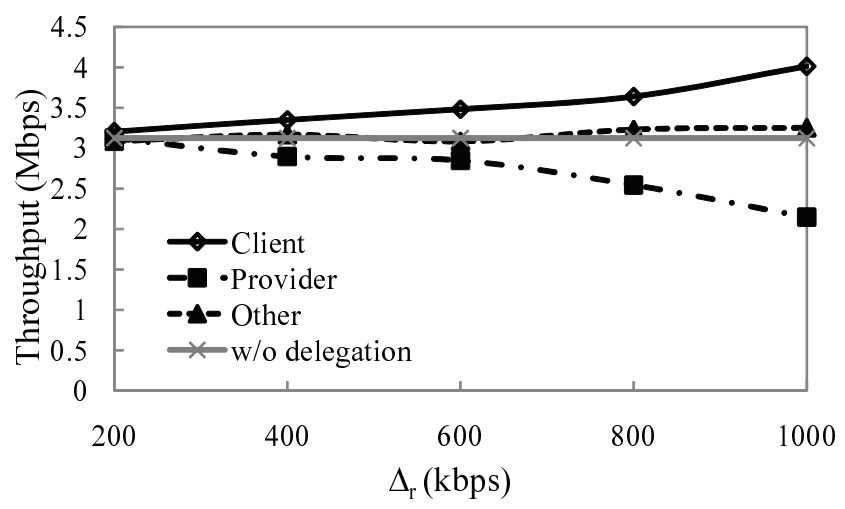

Figure 13 Throughput for each STA versus $\Delta_{r}$ with one additional TCP flow other than client and provider (other). $\Delta_{r}$ indicates the required additional throughput from the client. The model in Figure 9 was used.

while others' throughputs are not changed regardless of RTT. As in the above observations, our method enables the client to increase its throughput, which is always beneficial regardless of how much the increase is particularly when they download files or receive audio/video streams with progressive download as described in Section 1.

\section{Related work}

Our window-size delegation method in Section 3 enables an STA to delegate its throughputs to another STA in accordance with the required throughput without any effect on other STAs. Our method requires only a proxy server which is compliant with our method. To the best of our knowledge, any conventional methods cannot do this. In this section, we introduce several conventional methods as below.
Many flow level QoS control methods have been proposed including IntServ and DiffServ architectures $[2,3,9,10]$. In $[2,3,9]$, their approaches are basically to control bandwidths and/or delays of flows between edge routers. They can differentiate throughput and/or delays among flows, while they require replacements or modifications on edge routers, which are limited to their application range. On the other hand, it has been discussed how to prioritize throughputs for specific STAs with only modifications on a server [10]. In [10], when a server receives duplicate ACKs from prioritized STAs, the server decreases congestion window size of flows for other altruistic STAs instead of the prioritized STA's congestion window size. However, this method cannot ensure to increase a throughput of prioritized STA when the number of altruistic STAs is not satisfactorily large.

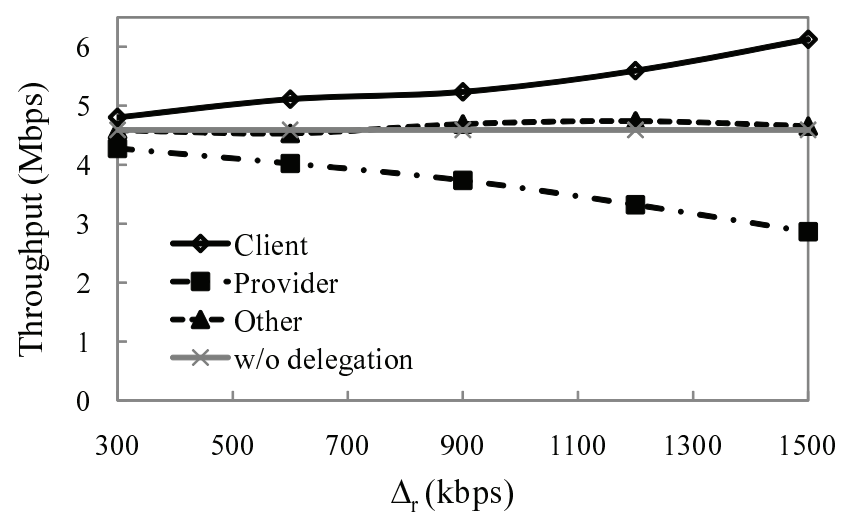

Figure 14 Average throughput of each STA versus $\Delta_{r}$ in single-AP network with provider, client, other STA. Bottlenecklink is IEEE $802.11 \mathrm{a}$ WLANs with physical transmission rate of $24 \mathrm{Mbps}$. 


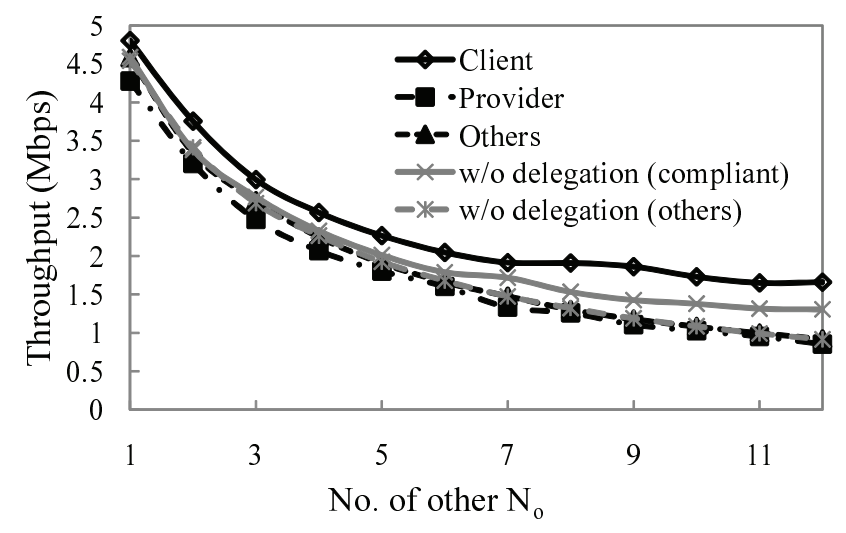

Figure 15 Average throughput of each STA versus $N_{o}$ in network with provider, client, and other $N_{o}$ STAs. Bottlenecklink was IEEE 802.11a WLAN with physical transmission rate of $24 \mathrm{Mbps}$. RTTs between AP and corresponding servers were identically $10 \mathrm{~ms}$.

On the other hand, in WLANs, MAC level QoS control methods also have been proposed including a QoS standard of WLANs called IEEE 802.11e [1]. The AP equipped with IEEE 802.11e can prioritize packets classified as specific traffic like video and voice and differentiate throughputs for them from the other traffic. However, it does make it without giving some effect on other STAs in the network especially when the network includes non-compliant STAs. Cooperation methods have been also discussed $[11,12]$ in WLANs. They discussed cooperation in packet forwarding, which can be also effective but is a different model from ours. Furthermore, most of the conventional cooperation methods in wireless networks including [11] and [12] were discussed only in the link or/and physical layer.

\section{Conclusion}

We proposed a TCP window-size delegation method for downlink TXOP Exchange in WLANs. In our method, a proxy server lets one station (the TXOP provider) delegate TCP window size to another station (the TXOP client) so that the provider delegates its TXOPs in CSMA/ CA to the client. Our method enables an STA to flexibly delegate TXOPs to another STA without adversely affecting the legacy STAs, which is confirmed by computer simulations. We also confirmed that our method requires no modification to legacy APs and STAs and needs only minimal modifications of the TCP functions of the proxy server.

We would like to mention that our method enables $N$-providers to delegate their window size to $M$-clients

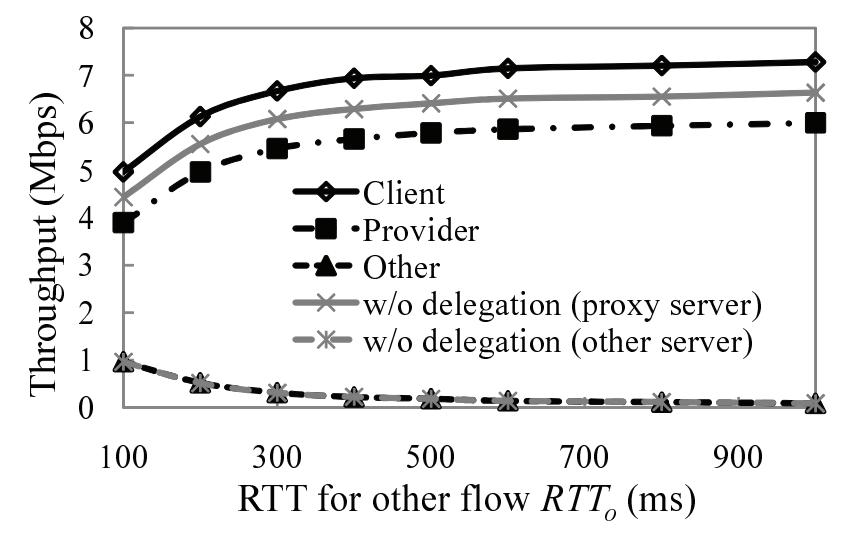

Figure 16 Average throughput of each STA versus RTT of flows for others. There are provider, client, and eight other STAs. RTTs between AP and corresponding server for provider and client were $10 \mathrm{~ms}$, while RTT for the other varied as $\mathrm{x}$ axis. Bottlenecklink was IEEE 802.11a WLAN with physical transmission rate of $24 \mathrm{Mbps}$. 
simultaneously, although, in this paper, we consider only a case where a provider delegates its window size to a client. We will observe the case where $N$-providers delegate their window size to $M$-clients in the future work. Future work also includes a design of a coordination algorithm that ensures fair incentives for cooperation between STAs.

\section{Acknowledgements}

This work is supported in part by the National Institute of Information and Communications Technology (NICT), Japan, under Early-concept Grants for Exploratory Research on New-generation Network.

\section{Author details}

'Graduate School of Informatics, Kyoto University, Kyoto, Japan ${ }^{2}$ Cybermedia Center, Osaka University, Osaka, Japan

\section{Competing interests}

The authors declare that they have no competing interests.

Received: 2 February 2011 Accepted: 11 August 2011

Published: 11 August 2011

\section{References}

1. IEEE Std 802.11e, Medium Access Control (MAC) Quality of Service Enhancement (Nov 2005)

2. R Braden, D Clark, S Shenker, Integrated Services in the Internet Architecture: An Overview, RFC 1633 (June 1994)

3. S Blake, D Black, M Carlson, E Davies, Z Wang, W Weiss, An Architecture for Differentiated Services, IETF RFC 2475 (Dec 1998)

4. T Nishio, R Shinkuma, T Takahashi, N Mandayam, TXOP Exchange: A Cooperative Resource Exchange Method in CSMA/CA, in IEICE General Conference, B-15-6 (March 2010)

5. T Nishio, R Shinkuma, T Takahashi, N Mandayam, Transmission-Opportunity Exchange for Cooperative Bandwith Allocation in Wireless Local Area Networks. IEICE Technical Report, MoMuC2010-3, 13-18 (May 2010)

6. T Nishio, R Shinkuma, T Takahashi, N Mandayam, A Coordination Algorithm for $\mathrm{N}$-node Cooperation Problem in Open-Resource Wireless Access Networks, in IEICE Communications Society Conference, BS-9-5 (Sept 2010)

7. QualNet, http://www.qualnet.com

8. J Padhye, V Firoiu, D Towsley, J Kurose, Modeling TCP throughput: a simple model and its empirical validation, in Proceedings of ACM SIGCOMM, 303-314 (Oct 1998)

9. M Guirguis, A Bestavros, I Matta, N Riga, G Diamant, Y Zhang, Providing soft bandwidth guarantees using elastic TCP-based tunnels, in International Symposium on Computers and Communications, Proceedings of ISCC 2004, 760-765 (July 2004)

10. J Valdez, M Guirguis, Liberating TCP: the free and the stunts, in International Conference on Networking, 2008, ICN 2008, $72-77$ (Apr 2008)

11. P Liu, Z Tao, S Narayanan, T Korakis, S Panwar, CoopMAC: a cooperative MAC for wireless LAN. IEEE J Sel Areas Commun. 25(2), 340-354 (2007)

12. P Bahl, R Chandra, P Lee, V Misra, J Padhye, D Rubenstein, Y Yu, Opportunistic use of client repeaters to improve performance of WLANs. IEEE/ACM Trans Netw. 17(4), 1160-1171 (2009)

doi:10.1186/1687-1499-2011-58

Cite this article as: Nishio et al:: TCP-based window-size delegation method for TXOP Exchange in wireless local area networks. EURASIP Journal on Wireless Communications and Networking 2011 2011:58.

\section{Submit your manuscript to a SpringerOpen ${ }^{\mathcal{O}}$ journal and benefit from:}

- Convenient online submission

- Rigorous peer review

- Immediate publication on acceptance

- Open access: articles freely available online

- High visibility within the field

- Retaining the copyright to your article

Submit your next manuscript at $>$ springeropen.com 